\title{
A leptonic/hadronic jet model for the low-mass microquasar XTE J1118+480
}

\author{
Gabriela S. Vila ${ }^{1}$ and Gustavo E. Romero ${ }^{1,2}$ \\ ${ }^{1}$ Instituto Argentino de Radioastronomía, (CCT La Plata - CONICET), C.C. N5 (1894), \\ Villa Elisa, Buenos Aires, Argentina \\ email: gvila@iar-conicet.gov.ar \\ ${ }^{2}$ Facultad de Ciencias Astronómicas y Geofísicas (FCAG, UNLP), Paseo del Bosque s/n \\ (1900), La Plata, Buenos Aires, Argentina \\ email: romero@iar-conicet.gov.ar
}

\begin{abstract}
We present a one-zone jet model that fits the data from simultaneous broadband radio-to-X-rays observations of XTE J1118+480. We calculate the radiative contribution to the non-thermal spectrum of both relativistic electrons and protons, as well as that from secondary muons, charged pions and electron-positron pairs produced at high-energy hadronic interactions. The distributions in energy of all the particle species are obtained taking into account the energy losses, injection, decay and escape from the emission region. We also include absorption effects on the emission spectrum due to photon-photon annihilation. Finally, we discuss the detectability of XTE J1118+480 at high energies with the present instruments according to the predictions of our model for the gamma-ray band.
\end{abstract}

Keywords. Radiation mechanisms: nonthermal, X-rays: individual (XTE J118+480).

\section{Introduction}

The source XTE J1118+480 is an X-ray binary located at a distance of $\sim 1.7 \mathrm{kpc}$ (Gelino et al. 2006) at high galactic latitudes $\left(b=+62.3^{\circ}\right)$. The mass of compact object is larger than $6 M_{\odot}$ (McClintock et al. 2000) being, therefore, a firm black hole candidate in the galactic halo. Although no relativistic jets have been directly imaged yet, the characteristics of the radio emission (e.g. Fender et al. 2001) strongly suggest that the source is a low-mass microquasar (LMMQ).

Several leptonic models have been proposed to explain the broadband radio-to-X-rays spectrum of XTE J1118+480 during the low-hard state (LHS), see for example Esin et al. (2001), Markoff et al. (2001), Yuan et al. (2005) and Maitra et al. (2009). In this work we present the application of a lepto-hadronic jet model to this source. As well as fitting the existing data, we intend to asses the possibility of gamma-ray emission from the jet due to different interactions processes involving relativistic particles.

\section{Model}

The jet model applied here is presented in detail in Vila \& Romero (2010) and references therein. We assume that the jet is injected at a distance $z_{0}=50 R_{\mathrm{g}}$ from the black hole and expands as a cone of semi aperture $\sim 6^{\circ}$. The outflow carries a $10 \%$ of the accretion power. The magnetic field at $z_{0}$ is $\sim 10^{6}-10^{7} \mathrm{G}$, and decays as $B \propto z^{-1.5}$. At a certain distance $\sim 10 z_{0}$ a fraction $q=0.1$ of the jet power is transferred to relativistic protons and leptons through diffusive shock acceleration. These particles are injected with a hard power-law spectrum $\propto E^{-1.5}$, and then suffer radiative and non-radiative 
losses until their energy distribution reaches the steady state. We calculate the electromagnetic emission from relativistic primary $\left(p\right.$ and $\left.e^{-}\right)$and secondary $\left(\pi^{ \pm}, \mu^{ \pm}\right.$and $e^{ \pm}$ pairs) particles due to several processes. Also added is the thermal emission spectrum from an standard accretion disc.

\section{Results and conclusions}

Figure 1 shows two least-squares fits to the LHS spectrum of XTE J1118+480 obtained with our model, corresponding to observations carried out during the outbursts of 2000 and 2005. Accretion rates of $0.1-0.2 \dot{M}_{\mathrm{Edd}}$ were necessary to achieve the observed Xray emission levels. The power injected in relativistic protons and leptons in the jet is approximately the same, with a ratio $L_{p} / L_{e}=1-3$.
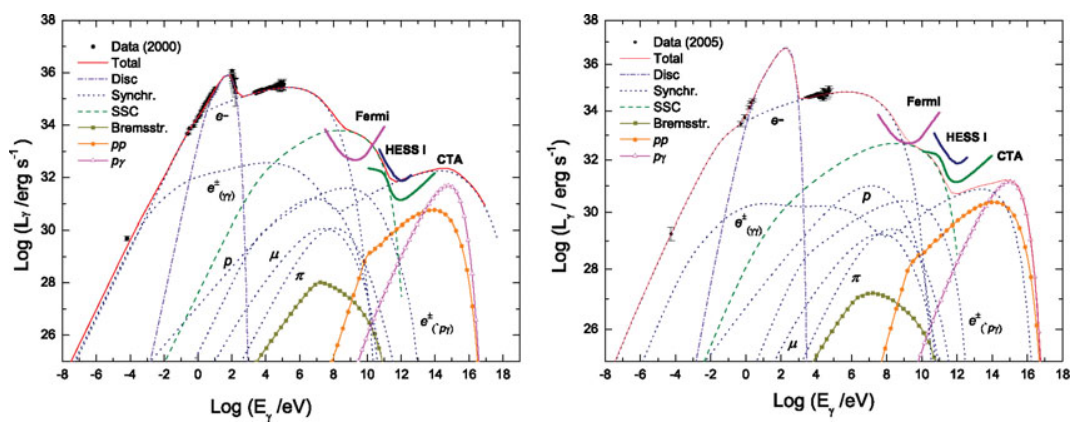

Figure 1. Model fits to the observed LHS spectrum of XTE J1118+480. The sensitivity curves of the Fermi satellite and the Cherenkov arrays HESS I and the future CTA are indicated.

The radio and X-ray data are well fitted with synchrotron emission from primary electrons. The direct hadronic contribution and electromagnetic radiation of secondary pions and muons are not significant. Synchrotron emission from $e^{ \pm}$pairs injected through $\gamma \gamma$ annihilation is relevant at energies above $\sim 0.1-1 \mathrm{TeV}$.

According to our results, the source would be detectable by Fermi in the GeV range with a soft spectrum. At $\mathrm{TeV}$ energies the synchrotron radiation of secondary pairs falls just below the sensitivity of HESS I (after 50 hours of exposure), but might be observed by the future CTA array. Our model also predicts that electron-positron pairs are produced at a significant rate. This is interesting in connection with the results of Weidenspointner et al. (2008), who found that the $0.511 \mathrm{MeV}$ annihilation line emission detected outside the galactic plane traces the asymmetric spatial distribution of low-mass X-ray binaries in the Galaxy (see, however, Bouchet et al. 2010).

\section{References}

Bouchet L. et al. 2010, ApJ, 720, 1772

Esin A. et al. 2001, ApJ, 555, 483

Fender R. et al. 2001, MNRAS, 322, L23-L27

Frontera F. et al. 2001, ApJ, 561, 1006

Gelino D. et al. 2006, ApJ, 642, 438

Maitra et al. 2009, MNRAS, 389, 1638

Vila G. S. \& Romero G. E. 2010, MNRAS, 403, 1457

Weidenspointner et al. 2008, Nature, 451, 159

Yuan F. et al. 2005, ApJ, 620, 905 